\title{
Rigid-Flexible Outer Sheath Model Using Slider Linkage Locking Mechanism and Air Pressure for Endoscopic Surgery
}

\author{
Akihiko Yagi $^{1}$, Kiyoshi Matsumiya ${ }^{1}$, Ken Masamune ${ }^{1}$, \\ Hongen $\mathrm{Liao}^{2}$, and Takeyoshi Dohi ${ }^{1}$ \\ ${ }^{1}$ Graduate School of Information Science and Techonology, The University of Tokyo, \\ 7-3-1 Hongo Bunkyo-ku, Tokyo, 133-8656, Japan \\ \{akihiko, mkiyo, masa\}@atre.t.u-tokyo.ac.jp, \\ takdohi@i.u-tokyo.ac.jp \\ ${ }^{2}$ Graduate School of Techonology, The University of Tokyo, \\ 7-3-1 Hongo Bunkyo-ku, Tokyo, 133-8656, Japan \\ liao@miki.pe.u-tokyo.ac.jp
}

\begin{abstract}
The objective of this paper is to develop an outer sheath for flexible endoscopic manipulators. This sheath can switch two states including flexible and rigid, and make a rigid curved path for inserting manipulators. The flexible mode can be curved into a required shape. The rigid mode can hold the shape of the sheath, and then keep the path for instruments. Through the managed path, the flexible manipulators become easy to reach the target. We proposed a serial multi joint model to realize the flexible mechanism. This model is composed of a set of frame units which are connected serially. Each unit can be rotated to a given angle around the center of the joint. We developed a sliderlink mechanism and a gear stopper controlled by air pressure for rigid mode. We designed and fabricated the prototype with a diameter of $16 \mathrm{~mm}$ and length of $290 \mathrm{~mm}$. The experiment showed that the device could be switched from the flexible mode to the rigid mode when the air pressure was over $150 \mathrm{kPa}$, and each joint could hold its angle against the maximum $400 \mathrm{mNm}$. The phantom experiment showed that the flexible devices are possible to transmit the wire tension to the endpoint of the manipulator without changing the curving shape with by the developed outer sheath device.
\end{abstract}

\section{Introduction}

Laparoscopic surgery has been widely performed in abdominal surgery. Surgeon can insert special instruments from small holes on the abdominal wall and perform operation after lifting the abdominal wall. To improve the performance and reduce the risks of this surgery, many researchers and engineers developed various instruments such as multi degrees-of-freedom (DOFs) bending manipulators [1-3] or Master-Slave type manipulators 4]. However, some problems remain unsolved. First, laparoscopic surgery requires wide space below the abdominal 
wall. Pneumoperitoneum is commonly used to lift the abdominal wall, but some complications caused by this method are reported. Second, although the laparoscopic surgery can approach the target in anterior area of the body, it is difficult to approach the target in deep, narrow area. To address these problems, instruments are required to be flexible to go into the body avoiding the critical areas. Several groups developed different flexible manipulators with wide curve. Ikuta et al. developed a micromanipulator to reach difficult area [5]. Moreover, other flexible manipulators using shape memory alloy (SMA) [] , or wire-driven mechanism were also developed[7]. However, flexible instruments can not be inserted easily in narrow space between the tissues or organs, and can not be stabilized completely when approaching target. In order to solve problem of flexible instruments, it is required to manage rigid path to insert flexible instruments in advance.

The purpose of this study is to develop an outer sheath to make an instrumental path in human body. This sheath is able to be any given shape, and to hold the shape against of the external force. Before inserting flexible instruments, the surgeon inserts the outer sheath through the narrow gap between the safety areas. After reaching to the target, the surgeon locks the shape, then the surgeon is able to insert flexible instruments easily through the planned path. In order to realize the switch of two states, flexible and rigid, we used multi-joint model for flexibility and slider-linkage mechanism and air pressure locking mechanism for rigidity. The engineering contribution of this paper is to devise a mechanism which can change its shape to be given shape and generate stiffness in the given shape. The clinically significance is to enable to manage rigid curved path for flexible instruments. Then, it becomes possible to approach to the target by less invasive way. This paper reports 1) a mechanism that enables to switch flexible mode and rigid mode, 2) a prototype of outer sheath and total system, and 3 ) evaluation experimental results about the stiffness and clinical applicability study using phantom and flexible device.

\section{Method}

The outer sheath that we try to develop is able to change its shape with a number of DOFs. To realize this function, the multi joint model is used 8 . The outer sheath consists of several pieces of short sheath. These sheath units are connected serially to make a long sheath. Each unit is able to be rotated several degrees around the joint. So this kind of outer sheath is possible to be changed to different (Fig. 11). To lock the shape and keep from the external force, we proposed slide-linkage locking mechanism. Each unit has a link, a slider, a stopper, and an air channel inside the instrument. The unit and the slider are connected by a link with pin joints. The slider moves in the slider channel of the adjacent unit in conjunction with the rotation of the unit. Sliders and the stoppers have gear cogs. When air pressure pushes the stopper, the stopper meshes the gear tooth of the slider. The rotation of the unit is locked after locking the slider, so the sheath gets rigid. When the air pressure is off, the spring attached to the stopper 


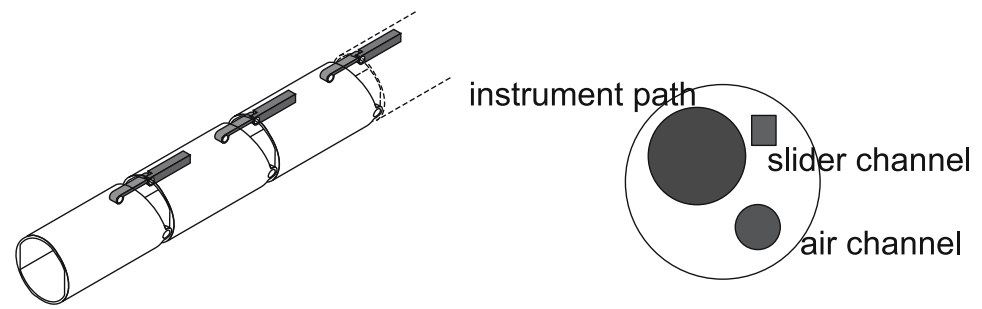

Fig. 1. The perspective view (left) and cross section image (right) of the outer sheath model: Our outer sheath consists of pieces of the sheath, link, and slider. Link and slider slide in slider channel and flexible tube passes through air channel. Instruments pass through the instrument path.

push the stopper back, and then the sheath is switched to the flexible mode (See Figure 21).

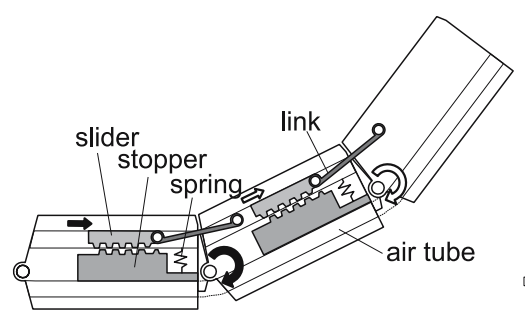

(a)

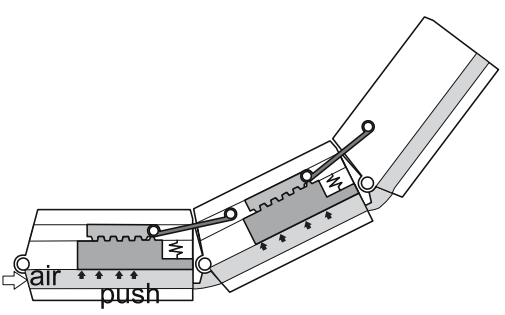

(b)

Fig. 2. Mechanism of having stiffness:When fluid channel is empty, the piece, link, and slider move in conjunction, and each pair moves independently (a). When the air pressure is add, the stopper moves up and mesh with the gear rack of the slider, then the shape of the sheath is locked (b).

\section{ProtoType}

We made a prototype using above mechanism(Fig. [3). Long units and short units are connected alternately, and two joints of each unit have different direction of rotation axis. Then the sheath is able to make cubic curve. The cross section of this sheath is circle diameter of $16 \mathrm{~mm}$. Diameter of the instrument path is $8 \mathrm{~mm}$. Polyvinyl chloride tube is attached in the inner guide tube of this hole, so the sheath enables instruments to go through the device with a diameter of less than $6 \mathrm{~mm}$. Diameter of the air channel is $3.6 \mathrm{~mm}$. The natural rubber tube is attached to transmit air pressure to the stopper. The length of long unit is $22 \mathrm{~mm}$, and that of short unit is $7 \mathrm{~mm}$. Total length is $290 \mathrm{~mm}$. Constituent material of the unit frames is aluminium. The links, sliders, and joint pins is made of stainless-steel. The total weight is $98 \mathrm{~g}$. 


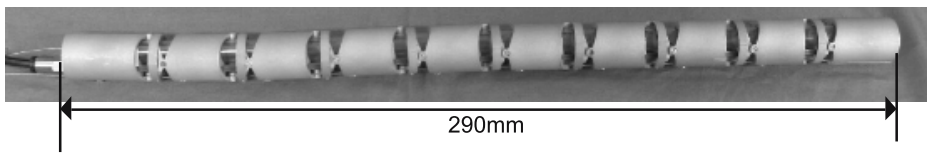

(a)

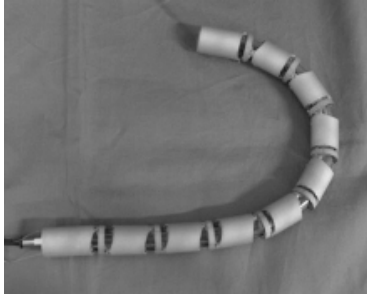

(b)

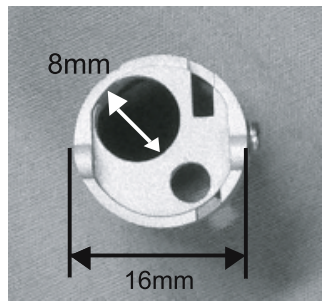

(c)

Fig. 3. The image of prototype: (a) shows when this outer sheath becomes straight. (b) shows that this out sheath is possible to curve and lock the shape. (c) shows the cross section surface.

The prototype has one pair of wire guides periodically spaced along its length. Two wires run through the wire guide eyelets, terminating at the endpoint. Through the wire tension, the sheath curves and change direction of the endpoint such as planar continuum manipulators 9 when the sheath is flexible. The wire is pulled by hand, and this wire tension supports to going into the sharp curved route and selects the planned route from branched route.

Figure 4 shows the system configuration. This system consists of the outer sheath device and air pressure controller. The controller switches to supply air pressure and controls the pressure. Air source is supplied from air supply port in operating room, gas cartridge, or simple air pump. This prototype the hand type switch is used, but any type, such as foot switch, is usable. This sheath is used under draping. In the future the wire tension form motors and motor drivers, then the outer sheath will be controlled from PC.

\section{Validation}

We evaluated the basic mechanical characteristic and clinical applicability. First we evaluated the relationship between the air pressure and the ability to keep the shape against external force (external torque). External force is added to the head of the sheath perpendicular to the direction of first unit (In this paper we call the pieces and joints sequential order form here.). Then, we measured the external force that rotates the first unit around the first joint, with a air pressure change from $0 \mathrm{kPa}$ to $400 \mathrm{kPa}$. Figure 5 shows two clear states switching on the certain border air pressure. When the air pressure is low, the external force is about $0 \mathrm{gf}$, this means the first piece rotated freely. When the pressure is high, the unit holds its angle even the external force is add. The border zone of the air pressure is from $130 \mathrm{kPa}$ to $150 \mathrm{kPa}$. 


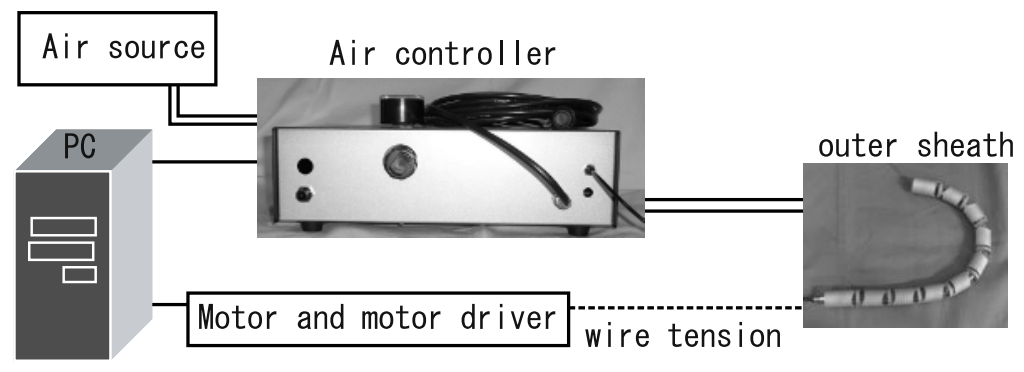

Fig. 4. The system of rigid-flexible outer sheath: The system consists of the outer sheath and air controller. Single lines are the connection of electric signals. Double lines are connection of air. Dashed line is the image of wire. Future, the wire tension will be controlled from motor and PC.

Next, we measured the external force by keeping the angle of each joint in the same condition about external force. The air pressure was set to $250 \mathrm{kPa}$. In this experiment the shape of the sheath was straight. The first joint and second joint keep their angle against over $5 \mathrm{~N}$, but third joint bends when the external force is $4.5 \mathrm{~N}$. Maximum force becomes lower when the distance between the joint and the forced point is long. As the result, the maximum torque should be $400 \mathrm{mNm}$.

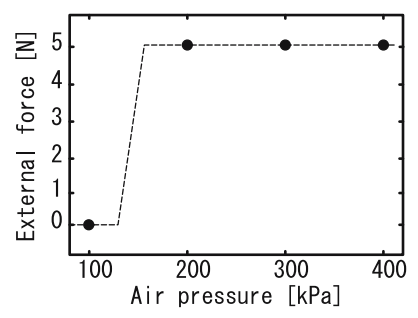

Fig. 5. Relation between rigidness and air pressure: Vertical axis is the external force under which first joint could hold its locked angle. The force was added perpendicular to the direction of the axis of the sheath. We tested the external force when the air pressure is added every $100 \mathrm{kPa}$, and every $10 \mathrm{kPa}$ between $100 \mathrm{kPa}$ and $200 \mathrm{kPa}$. When the air pressure is between $130 \mathrm{kPa}$ and $150 \mathrm{kPa}$, the rigidness is unknown.

Last, we evaluated clinical applicability by the phantom experiment. As the alternative of human soft tissues and gap between organs, narrow curved path was made and the silicone gel was filled around the path(Fig. 6a). In this experiment the path was the arc of which curvature radius is $10 \mathrm{~cm}$. When the outer sheath is inserted, it has to push the silicone wall to go through the path and reach to the target. In this experiment we assume that the sheath and manipulators go to the backside of the liver passing around liver and cholecyst (Fig. [6:), so the silicone is shaped like liver. As a flexible device, we used the laser 


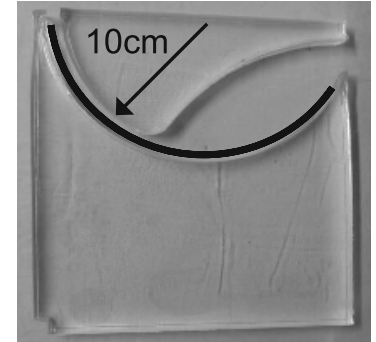

(a)

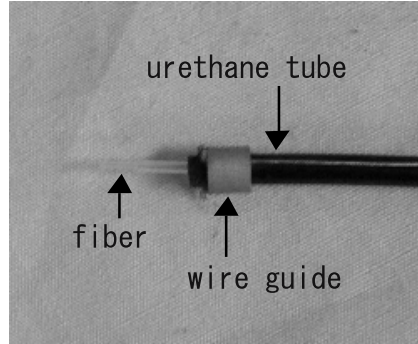

(b)

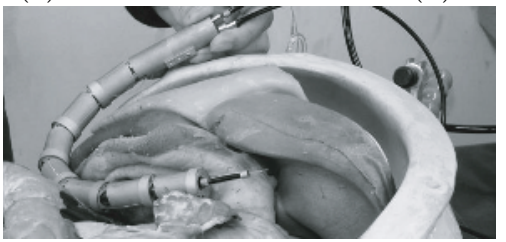

(c)

Fig. 6. Experiment condition: (a) curved course made by gelatin. The curveture radius is $10 \mathrm{~cm}$, and the thickness is $25 \mathrm{~mm}$. (b) insert material. The diameter of fiber core is $1 \mathrm{~mm}$. Urethane tube is $3 \mathrm{~mm}$, and that of wire guide is $4.5 \mathrm{~mm}$. (c) assumption of the phantom. This phantom assumes that the sheath goes through the gap around liver and cholecyst, and reaches to target on the backside liver.

fiber. The fiber(Standard-Lightguide, Dornier MedTech inc, German) is covered with urethane tube and wire guide device is attached periodically spaced. Two wires runs though with the eyelet of wire guide attachments. This fiber can be controlled by the difference of wire tension. The maximum diameter of the fiber device is $4.5 \mathrm{~mm}$ (Fig. 6b). The outer sheath wasn't able to go through the path only by pushing the sheath because the top of sheath was blocked by the silicone wall. However, with supporting the wire tension, it could go the path smoothly the path. (Fig. 7). After inserting the outer sheath device, the laser fiber device was inserted smoothly passing through the rigid path. When wire tension was add, the fiber device changed its direction of the tip without changing the whole shape of the device, so this manipulator could be controlled the direction of laser. The wire tension to bend the fiber was $18 \mathrm{~N}$.

\section{Discussion}

Experimental results show that slider linkage locking mechanism is sufficient to fix the shape. Laparoscopic manipulators can generate about $500 \mathrm{gf}(4.8 \mathrm{~N})$ force perpendicular to the direction of axis to lift up heavy organs in the body. The outer sheath keeps its shape against such force. Since our manipulators can go through the narrow gap between tissues, it is not necessary for the surgeon to move the organs. As for the air pressure, the outer sheath switches the pressure less than $200 \mathrm{kPa}$. It is not high pressure, so the air pressure may not damage 


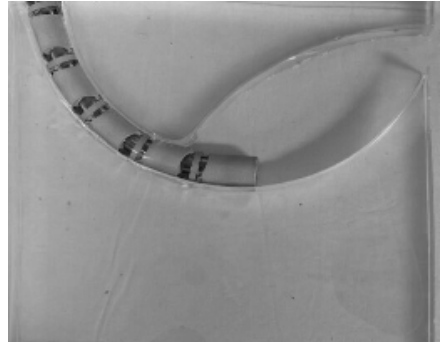

(a)

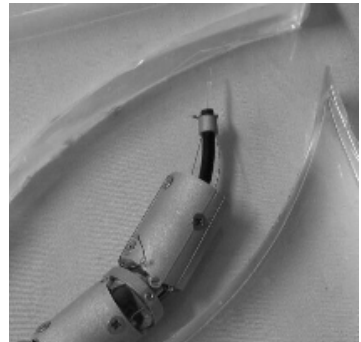

(b)

Fig. 7. The image of experiment: (a) shows that the outer sheath went through the path pushing silicone walls. (b) shows the endpoint of the sheath and laser fiber curved by wire tension.

the tissues around the sheath if the air leak, or burst. Then it can be used safely. From the phantom experiment, this sheath is considered to be able to go back side passing around the tissues. The arc whose radius is $10 \mathrm{~cm}$ is a little large path to go around small organs. The sheath isn't able to go large curvature route, or bend the head like bending manipulators. Then it is difficult to go around the small organs. However, it is sufficient to go between abdominal wall and tissues, or go thorugh the subxiphoid route. Wire tension is helpful to go the sharply curved route. In this prototype the tension affects to all joints, but the wire tension is helpful for only the first joint. By the mechanism to transmit the wire tension to first joint, the sheath will be easier to go narrow route. Using this sheath, the manipulators can be inserted smoothly, and transmit the wire tension to the endeffector. In phantom experiment we used a laser manipulator. We think that the gastroenterological endoscope is also useful combined with the sheath.

Next challenging problem is to detect the place of the endpoint of the sheath in human body, especially while inserting this sheath. X-ray is one effective way, but exposure problem occurs. Then smart way to detect the position is required.

We think that it become to be able the endoscopic surgery in deep, narrow area by the combination of our outer sheath and the wire-driven flexible manipulators. Our research is important step to make surgery less invasive.

\section{Conclusion}

We developed a rigid-flexible outer sheath that secures instrumental path for flexible device to make minimally invasive laparoscopic surgery. The mechanism to switch flexible mode and rigid mode using multi-joint model and slider-linkage mechanism is developed and evaluated. The air pressure and gear lock mechanisms enable a freedom shape locking. The results show that the outer sheath is able to bear about $5 \mathrm{~N}$ force on head, and $400 \mathrm{mNm}$ torque. Furthermore, flexible devices are possible to change the direction of its endpoint without changing 
the whole curving shape. Our mechanism that combined of the outer sheath and flexible manipulators is suitable to the laparoscopic surgery in deep, narrow area where the open surgery is necessary.

\section{Acknowledgment}

A part of this work is supported by Research Program for development of analysis, help, and alternation of human function "Research for the development of new surgical robot", administrated by Ministry of Health, Labour and Welfare of Japan.

\section{References}

1. Peirs, J., Reynaerts, D., Van Brussel, H.: A miniature manipulator for integration in a self-propelling endoscope. Sensors and Actuators A. 92 (2001) 343-349

2. Nakamura, R., Oura, T., Kobayashi, E., Sakuma, I., Dohi, T., Yahagi, N., Tsuji, T., Hashimoto, D., Shimada, M., Hashizume, M.: Multi-DOF Forceps Manipulator System for Laparoscopic Surgery - Mechanism miniaturized \& Evaluation of New Interface -. Proc. of 4th International Conference on Medical Image Computing and Computer-Assisted Intervention. (2001) 606-613

3. Yamashita, H., Hata, N., Kim, D., Hashizume, M., Dohi, T.: Handheld Laparoscopic Forceps Manipulator Using Multi-slider Linkage Mechanisms. Proc. of 7th International Conference on Medical Image Computing and Computer-Assisted Intervention. 2 (2004) 121-128

4. Abbou, C.C., Hoznek, A., Salomon, L., Olsson, L.E. Lobontiu, A., Saint, F., Cicco, A., Antiphon, P., Chopin, D.: Laparoscopic radical prostatectomy with a remote controlled robot. JOURNAL OF UROLOGY. 165 (2001) 1964-1966

5. Ikuta, K., Sasaki, K., Yamamoto, K., Shimada, T.: Remote Microsurgery System for Deep and Narrow Space - Development of New Surgical Procedure and Microrobotic Tool. Proc. of 5th International Conference on Medical Image Computing and Computer-Assisted Intervention. (2002) 163-172

6. Nakamura, Y., Matsui, A., Saito, T.: Shape Memory-Alloy Active Forceps for Laparoscpic Surgery. Proc. of the 1995 IEEE International Conference on Robot and Automation. (1995) 2320-2327

7. Simaan, N., Taylor, R., Flint, P.: High Dexterity Snake-Like Robotic Slaves for Minimally Invasive Telesurgery of the Upper Airway. Proc. of 7th International Conference on Medical Image Computing and Computer-Assisted Intervention. 2 (2004) $17-24$

8. Okamoto, J., Iida, M., Nambu, K., Fujie, M., Umezu, M.: Development of MultiDOF Brain Retract Manipulator with Safety Method. Proc. of the 2003 IEEE/RSJ International Conference on Intelligent Robots and Systems. 3 (2003) 2594-2599

9. Ian, A.D., Christophoer, D.R., Ian, D.W.: Large Deflection Dynamics and Control for Planar Continuum Robots. IEEE/ASME Transactions on Mechatoronics. 8(2) (2003) 299-307 